\title{
HACIA LA PUESTA EN MARCHA VIRTUAL DE CÉLULAS ROBOTIZADAS BASADA EN GEMELO DIGITAL
}

\author{
Darío Orive, Nagore Iriondo, Oskar Casquero, Marga Marcos. \\ Departamento de Ingeniería de Sistemas y Automática Universidad del País Vasco / Euskal Herriko Unibertsitatea \\ dario.orive@ehu.eus, nagore.iriondo@ehu.eus, oskar.casquero@ehu.eus, marga.marcos@ehu.eus
}

\begin{abstract}
Resumen
Uno de los retos de la Industry 4.0 es disponer de sistemas de fabricación más flexibles, que permitan fabricar productos personalizados, adaptados a la demanda de los clientes, así como reducir tiempos de desarrollo y producción. En ese sentido, la optimización de las fases de diseño, desarrollo y validación del sistema de control del proceso de fabricación es crucial. Actualmente ya se dispone de herramientas software que permiten realizar modelos virtuales de máquinas y líneas de producción, denominados "Gemelo digital" (Digital twin), así como herramientas software para realizar la "Puesta en marcha virtual" (Virtual Commissioning), que permiten probar en simulación el programa de control del proceso contra un gemelo digital del mismo. Este artículo presenta la forma de proceder para el desarrollo del gemelo digital de células robotizadas y su puesta en simulación con SiL (Software in the Loop) y HiL (Hardware in the Loop), con herramientas de la plataforma PLM de Siemens.
\end{abstract}

Palabras Clave: Industria 4.0, Gemelo Digital, Puesta en Marcha Virtual, Tecnomatix (Process Simulate), Sistemas de Automatización, Sistemas de Fabricación Flexibles.

\section{INTRODUCCIÓN}

Desde hace ya años estamos inmersos en la $4^{\mathrm{a}}$ Revolución Industrial, conocida como Industria 4.0 [3], cuyo objetivo es lograr que la industria sea capaz de aplicar conocimiento adquirido y ser capaz de responder ágilmente para adaptarse a un entorno cambiante. Para ello es necesario gestionar, de forma simultánea, un conjunto heterogéneo de aspectos y tecnologías que lo convierten en un problema muy complejo. No obstante, existen disponibles un conjunto de tecnologías y estándares y existen estudios que establecen pautas para lograrlo. Por ejemplo, en [4] se presenta un modelo de madurez que permite a las empresas afrontar este reto en seis fases. Las cuatro primeras son: digitalización global, comunicación (entendida como la capacidad de comunicar información relativa a cualquier activo de la empresa), visibilidad, refiriéndose a la capacidad de construir modelos de la empresa que representan su estado real a partir de dicha información y transparencia, es decir, permitir el acceso a los datos para crear nuevo conocimiento por agregación. Las dos últimas fases son la capacidad de predecir y la adaptabilidad, que permiten la toma de decisiones para adaptarse a cambios del contexto. Es en estas dos últimas fases donde entra en juego lo que se conoce como gemelo digital, representación virtual de la realidad a lo largo de las diferentes fases del ciclo de vida de un producto, desde la concepción o idea inicial, pasando por los diferentes procesos de fabricación, trazabilidad, distribución y venta, hasta el reciclado final del mismo.

En este artículo se proponen los pasos a seguir para construir el gemelo digital de una aplicación en la que intervienen robots, así como para la validación del sistema de control o puesta en marcha virtual con herramientas de la plataforma PLM de Siemens.

La estructura del artículo es la siguiente: El apartado 2 se dedica a presentar el Gemelo digital (Digital Twin) y algunas herramientas software que permiten desarrollar estos modelos. En el apartado 3 se discute la importancia de la puesta en marcha virtual (Virtual Commissioning), para probar y validar, el proyecto de control. El apartado 4 se presenta el caso de estudio de una célula robotizada para la fabricación de una parte de un calderín. El artículo finaliza con el apartado de conclusiones.

\section{GEMELO DIGITAL (Digital Twin)}

El gemelo digital de un proceso ofrece muchas posibilidades y beneficios en las tareas de ingeniería. Se pueden desarrollar desde modelos conceptuales, con ideas muy básicas de máquinas o procesos en las fases iniciales del proyecto, hasta modelos muy detallados de la máquina final fabricada. Con los modelos iniciales se pueden probar y validar funcionalidades que sin el modelo serían muy difíciles de probar. Pueden ayudar en la toma de decisiones y permiten realizar cambios en base a las pruebas realizadas.

La tecnología del gemelo digital puede ser utilizada desde el comienzo del desarrollo de un nuevo proceso automatizado, o también puede utilizarse 
para realizar mejoras o reformas de máquinas ya existentes, para adaptarlas y valorar su funcionalidad a las necesidades de cada momento [2].

En esta sección se presentan algunas de las herramientas existentes que soportan el ciclo de vida del producto. Concretamente, para obtener el gemelo digital o modelo de las máquinas del proceso, se dispone de diferentes herramientas en función de las características del proceso y de lo que se desee analizar. Así, la herramienta NX-MCD (Mechatronics Concept Designer) de la plataforma PLM de Siemens permite desarrollar gemelos digitales de máquinas formadas por accionamientos neumáticos, hidráulicos y eléctricos controlados por controladores con cierta inteligencia. Para obtener gemelos digitales de células muy automatizadas en las que intervienen robots industriales, la herramienta más adecuada es Tecnomatix PS (Process Simulate). Para analizar el flujo de los productos en la distribución en la planta (layout), la herramienta más adecuada el Tecnomatix (Plant Simulation). Para desarrollar gemelos digitales de proceso continuo, la herramienta más adecuada sería SIMIT. La forma de proceder en cada una de estas herramientas es diferente, pero todas ellas ofrecen un entorno de diseño gráfico y librerías que facilitan el desarrollo.

A continuación, se describe la forma de realizar el gemelo digital de un proceso industrial en el que intervienen robots industriales, mediante Tecnomatix (Process Simulate).

Se debe partir de una especificación funcional del proceso teniendo en cuenta la distribución espacial de las máquinas, almacenes, cintas de transporte, alimentadores de piezas, etc, en la planta, conocido como layout. En este tipo de instalaciones es frecuente encontrar, entre otros, dispositivos como:

- Robots Industriales que disponen de Unidad de Control inteligente (CPU controladora del robot), capaz de ejecutar el programa de control de estos dispositivos.

- Ejes lineales o giratorios, controlados en velocidad y/o posición desde unidades de control inteligentes (Variadores de frecuencia, Servos), capaces de realizar el control de los mismos.

- Otros dispositivos como prensas o atornilladores controlados también por sistemas inteligentes $\mathrm{y}$ programables.

Las herramientas de modelado de gemelos digitales deben permitir incluir este tipo de dispositivos en el gemelo, para obtener una representación virtual que se comporte lo más parecido posible al sistema real. De esta forma, los programas desarrollados para el gemelo digital, las pruebas de validación realizadas y los resultados obtenidos en simulación, representarán el comportamiento del sistema real.

\subsection{MODELADO DE ROBOTS}

Desde hace ya muchos años, el uso de robots industriales es práctica común en células de fabricación automatizadas.

Los pasos a seguir para incluir en el modelo del gemelo digital un robot industrial son los siguientes:

PASO 1: Selección del robot en función de la aplicación.

El primer paso es realizar la selección del robot en función de las características de la tarea o aplicación que va a realizar en el proceso real. En esta decisión hay muchas variables que hay que valorar, para lo cual hay que tener un gran conocimiento del proceso a automatizar, aunque este análisis no es objetivo de este artículo.

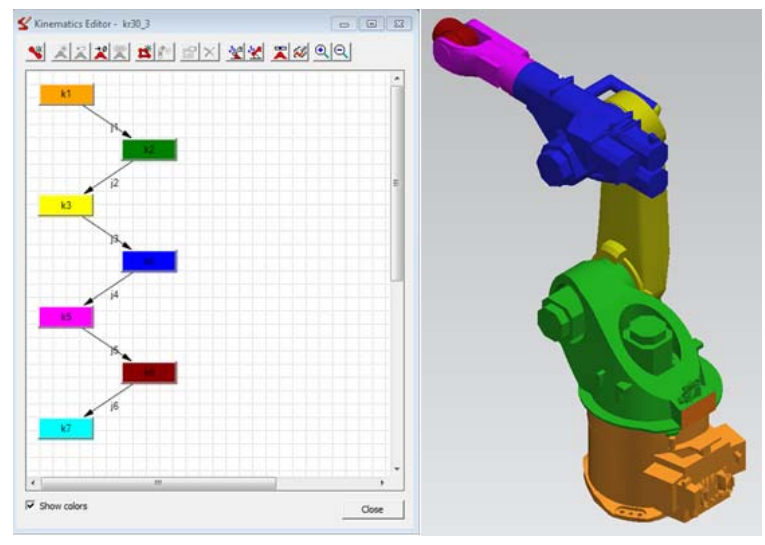

Figura 1.- Modelo de robot Kuka

PASO 2: Selección del robot en función de sus prestaciones para el gemelo digital.

Los fabricantes suelen ofrecer modelos de la estructura mecánica del robot, por un lado, y modelos de la unidad de control por otro. La variedad de situaciones que se pueden presentar son muchas. En modelos antiguos de robots, es difícil disponer de modelos mecánicos válidos para la simulación y de unidades de control que permitan incluir en el gemelo digital dicha funcionalidad. En modelos más modernos, es frecuente disponer, de forma gratuita, de los modelos mecánicos de los robots con la cinemática de su mecánica incluida. En relación con programas que simulen la funcionalidad de las Unidades de Control de los robots en el gemelo digital, a las cuales se las puede denominar Unidades de Control Virtuales, también hay cierta variedad; desde fabricantes que no las ofrecen o no disponen de las mismas, fabricantes que las facilitan de forma gratuita $y$ fabricantes que las facilitan con un 
determinado coste. En la figura 1 se puede ver cómo es el modelo mecánico de un robot de Kuka y el diagrama de la cinemática asociada a dicho modelo de robot. Este fabricante ofrece una Unidad de Control virtual, que es un fichero ejecutable. Para utilizarla hay que instalarla en la herramienta $y$ seleccionarla a la hora de realizar el modelo.

Del análisis de todas estas prestaciones se tomará la decisión final sobre la marca, modelo, unidad de control, garra, etc, del robot seleccionado.

PASO 3: Ubicación del robot en el escenario.

Una vez decidido de modelo de robot y se tienen disponibles los modelos suministrados por el fabricante, hay que proceder a ubicar el robot en el escenario del proceso.

El primer análisis que hay que hacer es el de los formatos o tipos de ficheros en los que se dispone el modelo del robot. Los entornos de modelado suelen exigir utilizar un tipo concreto, que dependerá de la herramienta concreta. En el caso de Tecnomatix (PS), el fichero tiene que ser *.jt. Lo primero que hay hacer es parametrizar el componente de tipo "Robot".

Para ubicar el robot en el escenario se puede hacer de varias formas; p.e. arrastrando el robot hasta el punto del escenario deseado o definiendo el sistema de coordenadas del robot respecto del sistemas de coordenadas general del escenario. También hay que asignarle el tipo de Unidad de control que se va a utilizar en el modelado (genérica o específica del fabricante). De esta selección dependerá el código generado en el desarrollo del gemelo.

Por último, hay que montar la herramienta (Mount tool) en el extremo del robot. Esto se realiza asociando el eje de coordenadas de la herramienta al TCP (Tool Center Point).

PASO 4: Desarrollar el gemelo digital del robot Una vez ubicado el modelo del robot en el escenario de la célula, hay que desarrollar en simulación todas las tareas que debe realizar el robot.

De forma similar a lo que se hace con equipos reales, el entorno de la herramienta de modelado con la que se está desarrollando el gemelo digital, debe permitir mover los ejes del robot hasta posicionarlo correctamente en los puntos adecuados del escenario. De todos estos movimientos, la herramienta de modelado genera, de forma transparente para el usuario, el programa de control de robot correspondiente a dicho movimiento, de forma que, se va generando el código del programa de control del robot, de forma paralela a la enseñanza de las tareas que tiene que realizar el robot en el proceso.
Como no todos los fabricantes ofrecen Unidades de Control virtuales que puedan ejecutar el código de control de los robots, es frecuente que las herramientas de modelado dispongan de una Unidad de Control genérica para poder ejecutar los movimientos del robot en simulación. La selección del tipo de Unidad de Control a utilizar en el gemelo digital (la genérica o la específica del robot) se realiza en los inicios del modelado de la aplicación. El código generado durante el modelado es diferente en función de en dónde se vaya a ejecutar. Si la Unidad de Control es la genérica, el código generado es genérico e independiente de la marca y tipo de robot que se esté utilizando. Este código no se podría cargar en la Unidad de Control del robot real. Si la Unidad de Control seleccionada es la específica del robot que se está utilizando, el código generado se corresponderá con el correspondiente a dicha Unidad de Control y, por lo tanto, el código una vez probado $\mathrm{y}$ validado en simulación en el gemelo digital, se pueden compilar y cargar directamente en la Unidad de Control real de robot.

\subsection{MODELADO DE EJES}

El modelado de máquinas con partes móviles que realizan desplazamientos de productos, como pueden ser cintas transportadoras, mesas giratorias, ejes, etc, se realiza siguiendo los pasos siguientes:

PASO 1: Se debe partir de un diseño 3D de la parte mecánica del elemento.

PASO 2: Parametrizar el tipo de componente de que se trata a partir de las opciones ofrecidas por la herramienta.

PASO 3: Ubicar el elemento en el escenario. Las formas de proceder, al igual que en el caso del robot, pueden ser por arrastre o por especificación de coordenadas.

PASO 4: Definición de la cinemática del elemento. Se deben agrupar aquellas partes o piezas del elemento que realizan el mismo movimiento (en NXMCD, estas agrupaciones se denominan Links). Posteriormente se debe definir el movimiento relativo de los grupos.

\subsection{MODELADO DE SENSORES}

Las herramientas de modelado permiten insertar sensores en el modelo del gemelo digital. Estos sensores deben ser los que se necesitan para desarrollar el proceso. Típicamente, permitirán detectar piezas o situaciones concretas en las que se encuentre el proceso. En general, los pasos a seguir son los siguientes: 
PASO 1: Seleccionar el tipo de sensor, de los soportados por la herramienta, adecuado a la funcionalidad que va a tener el mismo en la célula. Además, debe parametrizarse de acuerdo a las características de la célula.

PASO 2: Parametrizarlo a la situación concreta que va a tener el mismo en la célula (p.e. rango de detección). También es necesario definir con qué objetos tiene que activarse en la simulación.

PASO 3: Ubicar el sensor en el escenario. Las formas de proceder, igual que en el caso del robot, son dos: por arrastre o por especificación de coordenadas.

\subsection{CONCLUSIONES DEL PROCESO DE MODELADO}

El resultado final del proceso de modelado es disponer de un gemelo digital, desarrollado en un escenario idéntico al real, con los modelos de los dispositivos reales que tendrá el proceso real, incluyendo sensores y actuadores. Así mismo, se dispondrá en este momento del código de control de los robots, que se utilizará directamente en los robots del proceso real. Si en la definición del gemelo digital se hace uso de los mismos dispositivos que existirán en el proceso real, los resultados de simulación con gemelo digital serán de gran utilidad en la puesta en marcha del proceso real.

\section{PUESTA EN MARCHA VIRTUAL (Virtual Commissioning)}

Otra de las fases que aparecen como resultado de la digitalización es la denominada "Puesta en marcha virtual" (Virtual Commissioning) de procesos automatizados. Una de las tareas más estresantes y tediosas de la automatización de procesos es la puesta en marcha de máquinas y procesos in situ, con equipamiento real y, en la mayoría de los casos, sin prácticamente pruebas de validación previa. Esto tiene como consecuencia, en el mejor de los casos, importantes esfuerzos de programación en la fase final del proyecto, $y$ en el peor, posibles errores en la manipulación de los equipamientos, e incluso posibles daños o desperfectos en los mismos. La puesta en marcha virtual permite validar y corregir errores en simulación de una gran parte del sistema de control (hasta un 60\%) de forma que, la puesta en marcha final sobre el proceso real requiera un tiempo mucho más reducido, del orden de un $30 \%$ menos [1].

En esta sección se propone una metodología a seguir que se basa en las herramientas de la plataforma PLM de Siemens, en concreto Tecnomatix (PS) para modelado y SIMIT UNIT PN para la parte de simulación con Hardware in the Loop (HiL).
En el mundo real, para hacer una puesta en marcha se necesita disponer del proceso y del sistema de control. De la misma forma, en el mundo virtual, para hacer la puesta en marcha virtual, se necesita el proceso (en este caso un modelo del proceso o "gemelo digital" virtual) y un sistema de control, que en este caso puede ser virtual o real. Por lo tanto, a la hora de realizar esta tarea, se presentan varias alternativas cuya funcionalidad $\mathrm{y}$ prestaciones se describen a continuación.

\subsection{PUESTA EN MARCHA VIRTUAL CON SiL (Software in the Loop)}

PASO 1: Desarrollo del gemelo digital

Para realizar esta opción de puesta en marcha, se necesita disponer de un modelo virtual del proceso, desarrollado con las herramientas adecuadas. Este modelo puede tener un aspecto físico muy parecido al del proceso real o puede ser más conceptual. En la parte derecha de la figura 2 se presenta el modelo de una estación de una célula de manipulación, desarrollado en NX-MCD, que tiene un aspecto físico muy parecido al de la estación real.

\section{PASO 2: Inclusión de sensores y actuadores}

El gemelo digital que se va a utilizar para la puesta en marcha virtual debe disponer de entradas y salidas que le permitan interactuar con el sistema de control que lo va a controlar. Es decir, se debe tener en cuenta en el diseño del gemelo digital la inclusión de sensores, actuadores, y asociarlos a lo que en general se denomina "señales de control", para que el gemelo digital se pueda poner en simulación, evolucionar e intercambiar información, con el controlador (figu. 2)

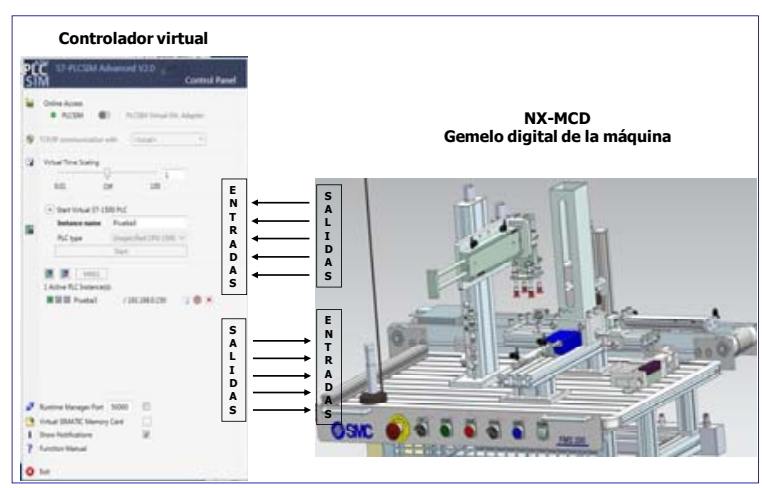

Figura 2. Puesta en marcha virtual con SiL

\section{PASO 3: Desarrollo del programa de control}

También se debe disponer de un controlador, en este caso, como es con SiL, el controlador deberá ser también virtual. En este controlador se ejecutará el programa de control con el que se desea controlar el proceso en simulación. En la Figura 2, se puede ver el Controlador Virtual, PLCsim Advanced, (parte izquierda de la figura). Se puede observar que el Controlador es una CPU virtual, es decir, un 
programa que ejecuta el programa de control del proceso. El programa de control estará desarrollado de la herramienta de ingeniería del fabricante del controlador, tendrá declaradas las E/S que utiliza para controlar el proceso y será cargado en la CPU virtual para su ejecución.

PASO 4: Relación de entradas-salidas del gemelo digital y del controlador virtual.

El siguiente paso es definir la relación entre las entradas-salidas del controlador y las salidas-entradas del gemelo digital. Las herramientas de modelado soportan interactuar con diferentes controladores virtuales y además, soportan diferentes formas de conectar las entradas/salidas. Entre todas las posibles, las más comunes son TCP/IP, OPC y OPC UA.

PASO 5: Puesta en marcha virtual

El proceso, representado por el gemelo digital, es otro programa que en ejecución se comportará de forma similar al proceso real. También es necesario disponer de entradas y salidas con las que se conecta al controlador. Cuando ambos programas se ejecutan, lo que puede realizarse en el mismo ordenador, se podrá observar la evolución del gemelo digital ante las órdenes recibidas desde el Controlador. De esta forma se pueden probar y validar los programas de control desarrollados.

Con la puesta en marcha virtual con SiL, sólo se valida el software del programa de control. Sin embargo, en la aplicación real será necesario, además, la configuración del hardware y las comunicaciones. Con SiL se utilizan las direcciones asignadas a las $\mathrm{E} / \mathrm{S}$, pero con independencia de que esas direcciones correspondan a una tarjeta ubicada en el Controlador o a una tarjeta ubicada en un dispositivo IO en Profinet IO. La ventaja que presenta esta forma de realizar la puesta en marcha es realizar con total independencia del hardware de los equipos de control reales, instalados en los armarios eléctricos y no disponibles para hacer validaciones y pruebas.

Entre sus limitaciones cabe destacar que no se pueden valorar, por ejemplo, tiempos de ciclo o tiempos de respuesta de ejecución del programa ya que las simulaciones son sobre CPUs genéricas de cada gama.

\subsection{PUESTA EN MARCHA VIRTUAL CON HiL (Hardware in the Loop)}

Antes de describir la simulación con HiL, indicar que, en general, un proyecto de automatización tiene como mínimo una parte de configuración del hardware de los controladores y de las E/S distribuidas a través de bus de campo. También suele haber, comunicaciones entre controladores, así como hacia otros sistemas de nivel superior. Por último, el proyecto tendrá el programa de control del proceso. Por lo tanto, se puede concluir que un proyecto de automatización tiene hardware, comunicaciones y software.

En esta segunda puesta en marcha virtual, se utilizan controladores reales (PLCs, PCs, ...), y gemelos digitales para representar el proceso. El gemelo digital se ejecuta en un PC y el programa de control desarrollado en la herramienta de ingeniería correspondiente al fabricante del controlador, en la CPU de un PLC real. Por lo tanto, el demostrador del que habría que disponer es el indicado en la figura 3. La forma de proceder es la siguiente.

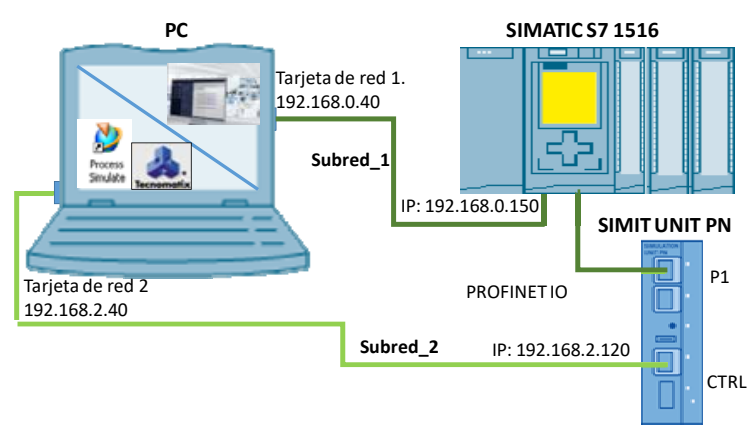

Figura 3. Puesta en marcha virtual con HiL

PASO 1: Desarrollo del gemelo digital.

Para el desarrollo del gemelo digital hay que proceder de igual forma que en el apartado 3.1. El gemelo digital generado es válido para la puesta en marcha virtual con SiL y HiL.

PASO 2: Montaje del demostrador.

Como se puede observar en la figura 3 hay que disponer de un controlador real y la tarjeta SIMIT UNIT PN. Hay que fijar todas las direcciones IP para configurar dos subredes diferentes. En el PC hay que disponer de las herramientas software para el desarrollo del gemelo digital y para el desarrollo del proyecto de control.

PASO 3: Puesta en servicio del controlador.

Si se parte de un proyecto de automatización con las tres partes indicadas en el aparado 3.2, (hardware, comunicaciones y software), y en esta situación, el proyecto de control se carga en un controlador real (PLC), al arrancar la ejecución del programa va a intentar comunicarse con los dispositivos IO configurados en el proyecto para acceder a la información de las E/S de los mismos. Como dichos dispositivos IO no existen en la realidad, la reacción de la CPU es pasar a stop, de forma que no ejecuta el programa de control y no se podría hacer ningún tipo de validación.

Para solventar este importante inconveniente, es necesario utilizar la tarjeta SIMIT UNIT PN, que como se indica en la Figura 3, soporta una comunicación Profinet IO con el controlador. En esta 
tarjeta hay que cargar la información correspondiente a la configuración de los dispositivos IO distribuidos, que se obtiene mediante la exportación de una parte del proyecto a un fichero, el cual, posteriormente se cargará en la tarjeta SIMIT UNIT PN. Esta tarjeta, con la información del hardware, emula la existencia de los dispositivos IO existentes y configurados en el proyecto, de forma que, cuando arranque el controlador encuentre la funcionalidad de dichos dispositivos IO en la tarjeta. Una vez configurada la tarjeta SIMIT UNIT PN, hay que cargar el proyecto de control en el controlador.

PASO 4: Relación de entradas-salidas del gemelo digital y del controlador real.

Para que el programa de control ejecutándose en el PLC real y el gemelo digital ejecutándose en el PC interaccionen, es decir, intercambien información de $\mathrm{E} / \mathrm{S}$, es necesario realizar el intercambio de información de las entradas-salidas de los dispositivos IO simulados en la tarjeta SIMIT UNIT PN con las salidas-entradas del gemelo digital del proceso ejecutándose en el PC. Dicho intercambio de información es realizado por la segunda subred establecida entre la SIMIT UNIT PN y el PC.

PASO 5: Puesta en marcha virtual con HiL.

Partiendo de un proyecto que conste de las tres partes indicadas en el aparatado 3.2, además del software de proyecto de control, como en el caso de SiL, se puede validar parte del hardware y simular las comunicaciones relacionadas con las E/S distribuidas. También se puede observar el tiempo de ejecución del programa de control en la CPU real, hacer ajustes con datos de ejecución en una CPU real, así como validar la parte del HMI.

Con HiL se pueden realizar muchas pruebas $\mathrm{y}$ validaciones parciales del proyecto, ya con cierto grado de acabado, sin necesidad de disponer del cableado real del sistema de control.

Esta forma de proceder presenta dos inconvenientes; tener que disponer de la CPU de un PLC real y tener que disponer de la tarjeta SIMIT UNIT PN, lo cual representa un coste importante, para poder realizar la puesta en marcha virtual.

\subsection{CONCLUSIONES DEL PROCESO DE PUESTA EN MARCHA}

La puesta en marcha de un proceso es una fase muy importante en el desarrollo de un proyecto de automatización. La precisión con la que se realice, va a depender el buen o mal funcionamiento del proceso. En muchos casos esta fase no se realiza todo lo bien que debe hacerse por diversas circunstancias. Las tecnologías actuales permiten simulaciones, que reducen el tiempo de puesta en marcha final.

\section{CASO DE ESTUDIO}

Algunas de las tecnologías y herramientas presentadas para el desarrollo de gemelos digitales y puesta en marcha virtual, han sido utilizadas para el desarrollo de una célula soldadura robotizada, para la fabricación de una de las partes de un calderín.

\subsection{DESCRIPCIÓN DE LA CÉLULA}

Especificaciones de la célula: El calderín de un calentador de agua caliente sanitaria es de chapa metálica y está formado por una superficie cilíndrica y dos tapas en los extremos del cilindro. El objetivo de la célula es automatizar la fabricación de la tapa inferior del calderín, formada por una chapa de forma circular, con dos orificios, uno para la entrada de agua fría y otro para la salida de agua caliente.

Escenario: El escenario o distribución de las máquinas que intervienen en el proceso es el indicado en la figura 4. Como se puede observar, hay una cinta transportadora por la que llegan las bases de forma circular, hay otra cinta por la se evacuan las piezas producidas y hay un alimentador de los tubos que hay que soldar en la base. Se han dispuesto en el escenario un robot de manipulación y un robot de soldadura. También se ha dispuesto una mesa giratoria sobre la que se realizan las operaciones.

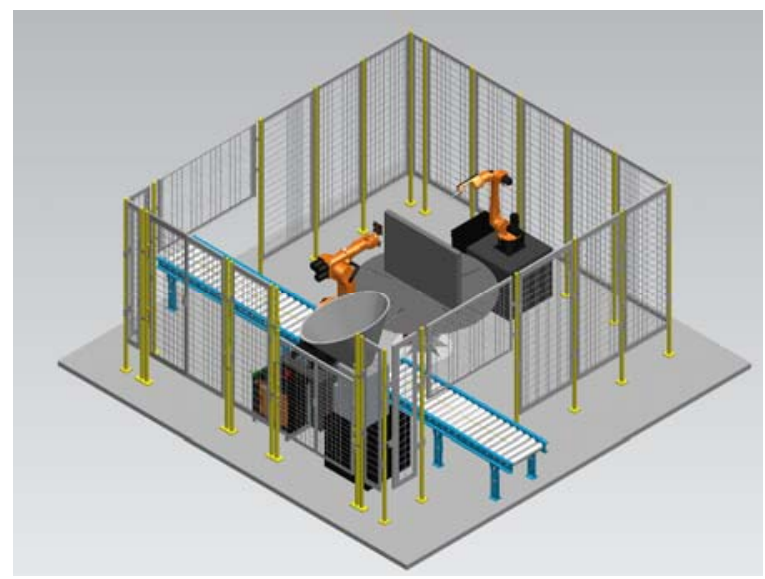

Figura 4: Escenario de la célula de soldadura

Cintas de alimentación y evacuación: La cinta de alimentación introduce en la célula las bases procesadas previamente. Es de rodillos y está accionada por un motor. La cinta de evacuación retira de la célula las bases una vez procesadas, también es de rodillos, y es accionada por un motor.

Alimentador de piezas de entrada y salida de agua: Consisten en dos tubos mecanizados previamente que hay que soldar en los orificios realizados en la base del calderín. Se ha dispuesto un alimentador para el posicionamiento correcto de los tubos. 
Robots: En la célula se realizan dos operaciones; una de manipulación y otra de soldadura. Se han valorado diferentes modelos y marcas de robots, y se han seleccionado dos robots de la marca KUKA, de 6 grados de libertad, uno para cada tipo de operación. Un factor muy importante en la decisión ha sido los modelos, tanto de la estructura mecánica como de la Unidad de Control, que ofrecía dicho fabricante.

Mesa de montaje y soldadura: Es una mesa giratoria, accionada mediante motor asíncrono asíncrono, sobre la que se realizan simultáneamente dos operaciones con los robots. El robot de manipulación realiza dos operaciones; retirar la base procesada en la cinta de evacuación y la operación de posicionado de la base sobre la mesa y el montaje de los tubos sobre la base. El robot de soldadura, realiza la soldadura de los dos tubos sobre la base. La mesa realiza giros de $180^{\circ}$ para presentar los componentes de la base, bien al robot de manipulación o, bien al robot de soldadura.

\subsection{GEMELO DIGITAL DE LA CÉLULA}

Entorno de desarrollo. Herramientas: Para el desarrollo del gemelo digital de la célula se han utilizado herramientas de la plataforma PLM de Siemens, en concreto, de la plataforma Tecnomatix, la herramienta denominada Process Simulate (PS).

Gemelo digital de las cintas transportadoras: Se han definido como "tipo de componente" conveyor. A continuación, se asignan los movimientos a la pieza.

Gemelo digital del alimentador de tubos: Se ha diseñado un modelo sencillo para simular las funciones de un alimentador por vibración.

Gemelo digital de las aplicaciones de los robots: Los modelos mecánicos y cinemáticos de los robots son los facilitados por KUKA. Se han ubicado en el escenario utilizando las funcionalidades que ofrece Tecnomatix (PS), definiéndoles de "tipo de componente" Robot. En el caso del robot de manipulación se ha diseñado una garra de dos pinzas, una para manipular los tubos y otra para la base.

Una vez ubicadas las máquinas en el escenario y analizadas las operaciones que tiene que realizar cada robot, se han diseñado y realizado los programas de los robots, 4 para el robot de manipulación y 2 para el robot de soldadura. Se han organizados adecuadamente para la selección de su ejecución desde el controlador de la célula (PLC). En estas tareas de modelado se ha obtenido código para la CPU virtual de los robots del fabricante.

Gemelo digital de la mesa giratoria: Se ha partido de realizar el modelo CAD 3D de la estructura mecánica en NX. Se ha insertado dentro de la herramienta Tecnomatix (PS) y se la ha definido como "tipo de componente" "Work table". Se la ha dotado de la cinemática definiendo un giro de $180^{\circ}$ alrededor del eje central de revolución de la mesa. (figura 5)

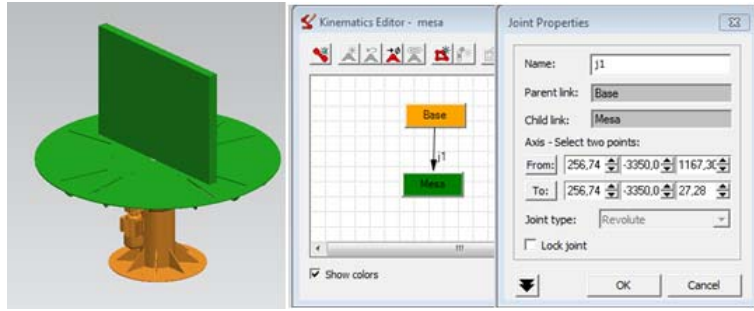

Figura 5. Modelo de la mesa giratoria

Con todos estos componentes convenientemente relacionados en tecnomatix (PS), se han podido realizar simulaciones para validar cinemáticas, posibles colisiones entre elementos de la célula, ...

\subsection{PROYECTO DE CONTROL DEL PLC}

El controlador de la célula es un PLC SIMATIC S7 1516 de Siemens. Además de la CPU con módulos de E/S, el sistema de control tiene una red Profinet IO a la que está conectado un dispositivo IO y las dos Unidades de Control de los robots. A través de las E/S del dispositivo IO se realiza el control de los motores de las cintas y mesa giratoria (ver figura 6)

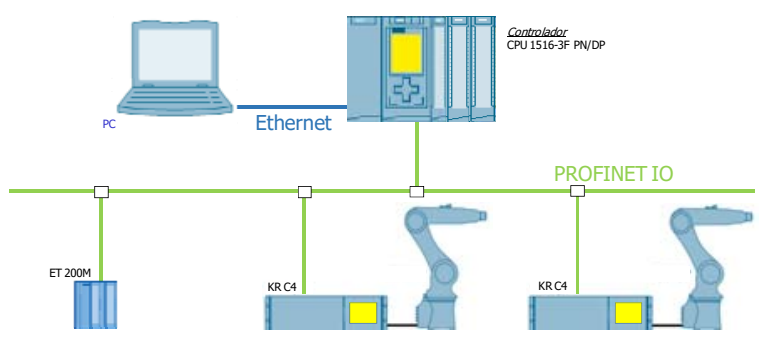

Figura 6. Sistema de control de la célula

Las Unidades de Control de los robots están en Profinet IO. Para desarrollar el proyecto de control en TIA Portal se han tenido que instalar los ficheros *.gsdml de ambas CPUs, facilitados por el fabricante. Con las Unidades de Control de los robots se intercambia, a través de la red, 40 bytes de entrada y 40 bytes de salida. Este tráfico de información es el que permite realizar el control de los robots desde el programa del PLC y poder realizar la puesta en marcha virtual de la célula con HiL, actuando sobre los programas de los robots.

El programa de control resumido de la célula es un grafcet con las diferentes tareas y el orden de ejecución de las mismas (ver figura 7). 


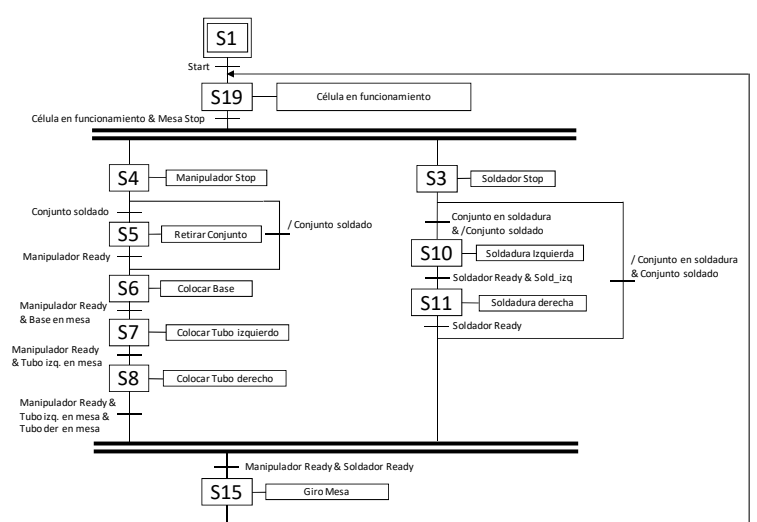

Figura 7. Secuencia simplificada del sistema de control de la célula

\subsection{PUESTA EN MARCHA VIRTUAL CON HiL}

En el caso de estudio se ha utilizado el demostrador de la figura 3.

El hardware utilizado es un PC con dos tarjetas de red Ethernet, un PLC SIMATIC S7 1516, una tarjeta SIMIT UNIT PN conectados en dos subredes. En cuanto al software, se ha dispuesto de la herramienta de ingeniería TIA Portal V15.1, Tecnomatix (PS) V14.1 y la herramienta Simulation Unit V 9.1, que permite realizar la carga del hardware de los dispositivos IO en la tarjeta SIMIT UNIT PN y el mapeado de las entradas-salidas del programa de control con las señales en Tecnomatix (PS). La tarjeta SIMIT UNIT PN simula la funcionalidad de los dispositivos IO del proyecto TIA Portal.

En una de las subredes están un puerto del PC, el PLC y el puerto P1 de la tarjeta SIMIT UNIT PN. Por ella se transfiere información de control con Profinet IO. En la segunda subred están el segundo puerto del PC y el puerto CTRL del la tarjeta SIMIT UNIT PN. Por esta subred intercambian información los dispositivos IO simulados en la tarjeta SIMIT UNIT PN y el gemelo digital en Tecnomatix (PS).

\section{CONCLUSIONES}

En el artículo de presenta la metodología para el desarrollo modelos de células robotizadas, denominados "gemelos digitales" y la forma de realizar la "puesta en marcha virtual" utilizando estos modelos. Todo ello en entornos de la plataforma PLM de Siemens.

\section{Agradecimientos}

Este trabajo está financiado por MCIU/AEI/FEDER, UE (proyecto RTI2018-096116-B-I00), GV/EJ (proyectos IT1324-19 y KK-2019-00095-DIGITAL).

\section{TOWARDS THE VIRTUAL COMMISSIONING OF ROBOTIC CELLS BASED ON DIGITAL TWIN}

\begin{abstract}
One of the challenges of Industry 4.0 is to have more flexible systems, which allow to manufacture more personalized products, adapted to customer demand, as well as reducing development and production times. In this sense, the optimization of the design, development and validation phases of the process control system is crucial. Currently, there are software tools to develop virtual models of machines and production lines, called "Digital Twin", as well as software tools to perform the "Virtual Commissioning", which allow simulation testing of the process control program against a digital twin instead of the real process. This paper presents, on the one hand, the steps to follow in order to develop digital twins of robotic cells, and on the other hand, how it can be simulated with both SiL and HiL using tools from Siemens PLM platform.
\end{abstract}

Keywords: Industry 4.0, Digital Twin, Virtual Commissioning, Tecnomatix (Process Simulate), Automation System, Flexible manufacturing systems

\section{Referencias:}

[1] Ayani, M., Ganebäck, M., Ng, A. H. C. (2018) "Digital Twin: Applying emulation for machine reconditioning". 51st CIRP Conference on Manufacturing Systems. Sweden, vol. 72, pp. 243-248.

[2] Negri, E., Fumagalli, L., Macchi, M. (2017) “ $A$ review of the roles of Digital Twin in CPSbased production systems". 27th International Conference on Flexible Automation and Intelligence Manufacturing, FAIM2017.

[3] Plattform Industrie 4.0, (2017) "What is Industrie 4.0?," pp. 4-5.

[4] Schuh, G., Anderl, R., Gausemeier J., ten Hompel, M., Wahlster, W. (Eds.), (2017) "Industrie 4.0 Maturity Index. Managing the Digital Transformation of Companies" (acatech STUDY), Munich: Herbert Utz Verlag.

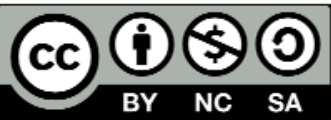

(C) 2019 by the authors. Submitted for possible open access publication under the terms and conditions of the Creative Commons Attribution CC BY-NC-SA 4.0 license (https://creativecommons.org/licenses/bync-sa/4.0/deed.es). 